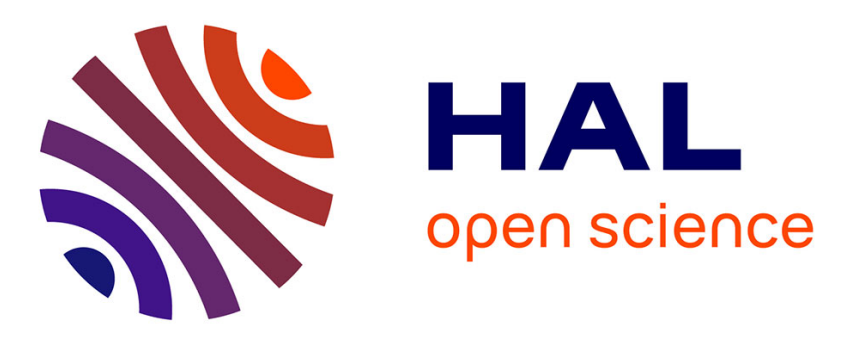

\title{
Diffusion MRI Anisotropy: Modeling, Analysis and Interpretation
}

Rutger H.J. Fick, Marco Pizzolato, Demian Wassermann, Rachid Deriche

\section{To cite this version:}

Rutger H.J. Fick, Marco Pizzolato, Demian Wassermann, Rachid Deriche. Diffusion MRI Anisotropy: Modeling, Analysis and Interpretation. 2017. hal-01468675

\section{HAL Id: hal-01468675 \\ https://hal.science/hal-01468675}

Preprint submitted on 23 Feb 2017

HAL is a multi-disciplinary open access archive for the deposit and dissemination of scientific research documents, whether they are published or not. The documents may come from teaching and research institutions in France or abroad, or from public or private research centers.
L'archive ouverte pluridisciplinaire HAL, est destinée au dépôt et à la diffusion de documents scientifiques de niveau recherche, publiés ou non, émanant des établissements d'enseignement et de recherche français ou étrangers, des laboratoires publics ou privés. 


\title{
Diffusion MRI Anisotropy: Modeling, Analysis and Interpretation
}

\author{
Rutger H.J. Fick, Marco Pizzolato, Demian Wassermann and Rachid Deriche
}

\begin{abstract}
The micro-architecture of brain tissue obstructs the movement of diffusing water molecules, causing tissue-dependent, often anisotropic diffusion profiles. In diffusion MRI (dMRI), the relation between brain tissue structure and diffusion anisotropy is studied using oriented diffusion gradients, resulting in tissueand orientation-dependent diffusion-weighted images (DWIs). Over time, various methods have been proposed that summarize these DWIs, that can be measured at different orientations, gradient strengths and diffusion times into one "diffusion anisotropy" measure. This book chapter is dedicated to understanding the similarities and differences between the diffusion anisotropy metrics that different methods estimate. We first discuss the physical interpretation of diffusion anisotropy in terms of the diffusion properties around nervous tissue. We then explain how DWIs are influenced by diffusion anisotropy and the parameters of the dMRI acquisition itself. We then go through the state-of-the-art of signal-based and multi-compartmentbased dMRI methods that estimate diffusion anisotropy-related methods, focusing on their limitations and applications. We finally discuss confounding factors in the estimation of diffusion anisotropy and current challenges.
\end{abstract}

Rutger H.J. Fick

Université Côte d'Azur, Inria, Athena Project Team, France, e-mail: rutger.fick@inria.fr

Marco Pizzolato

Université Côte d'Azur, Inria, Athena Project Team, France e-mail: marco.pizzolato@inria.fr

Demian Wassermann

Université Côte d'Azur, Inria, Athena Project Team, France e-mail: demian. wassermann@inria. fr

Rachid Deriche

Université Côte d'Azur, Inria, Athena Project Team, France e-mail: rachid.deriche@inria.fr 


\section{Introduction}

In brain imaging, diffusion anisotropy is a manifestation of tissues obstructing the otherwise free diffusion of water molecules. Brain tissues with different structural make-ups, e.g. healthy or diseased, influence the diffusion differently [Moseley et al., 1990, Beaulieu, 2002]. Relating the observed diffusion with the underlying tissue structure has been one of diffusion MRI's (dMRI's) main challenges. This challenge can be seen as a variant of the work Can One Hear The Shape of a Drum by Kac [1966]. Basser et al. [1994] were the first to determine the voxel-wise orientational dependence of diffusion in biological tissue by fitting a tensor to the signals of non-collinearly oriented diffusion gradients [Tanner and Stejskal, 1968]. For the first time, this representation made it possible to describe both the tissue orientation and the "coherence" of the underlying tissue by means of rotationally-invariant indices such as the Fractional Anisotropy (FA) [Basser, 1995]. Since then, a plethora of dMRI models have been proposed to more accurately relate tissue properties to the measured signal by using less or different assumptions on the tissue configuration or increasing the requirements of the signal acquisition [see e.g. Behrens et al, 2003, Tuch, 2004, Wedeen et al., 2005, Assaf et al., 2008, 2004, Alexander et al., 2010, Özarslan et al., 2013, Kaden et al., 2015, 2016].

This chapter is meant as a review of current methods that either directly estimate diffusion anisotropy measures or anisotropy-related tissue properties. We restrict ourselves to diffusion anisotropy a property of the overall diffusion signal (such as FA); anisotropy as a property of the dispersion of diffusion micro-environments; and anisotropy as a property of the micro-environment itself. In the latter two cases, we view a micro-environment as the diffusion profile of a single axon and its immediate surroundings. Using this definition, the signal measured from a bundle of axons can be seen as an ensemble of micro-environments, each having their own orientation and signal contribution to the overall signal. As an example of a method that estimates this micro-environment dispersion, we include the Neurite Orientation Dispersion and Density Imaging (NODDI) [Zhang et al., 2012]. Describing anisotropy as a property of one micro-environment, we include the Spherical Mean Technique [Kaden et al., 2015]. Finally, as diffusion anisotropy is a consequence of diffusion restriction and hindrance, we will put special emphasis on the influence and modeling of diffusion time. This angle of approach is timely, as STimulated Echo Acquisition Mode (STEAM) pulse sequences have recently allowed the in-vivo exploration of long diffusion times [De Santis et al., 2016, Fieremans et al., 2016], verifying the existence of time-dependent diffusion even at low bvalues [Fieremans et al., 2016].

The structure of this chapter is as follows: In Section 2, we first provide a physical interpretation on what diffusion anisotropy is. In Section 3 we clarify the mechanics of a standard Pulsed Gradient Spin Echo (PGSE) sequence and how the measured signal is influenced by the tissue. In Section 4, we then describe the most relevant techniques that have been proposed to estimate and interpret diffusion anisotropy. We describe the differences between different signal-based anisotropy measures and relate them to axon dispersion-related metrics. We then discuss the time-dependence 
of anisotropy measures in Section 5. In this last section we also go into the diffusiontime-dependence of diffusion restriction in the extra-axonal space [Novikov et al., 2014, Burcaw et al., 2015]. Finally, we discuss challenges and confounding issues that these methods face in Section 6.

\section{Diffusion Anisotropy: The Phenomenon}

The characteristics of diffusion anisotropy in the brain depend on how the diffusion process is restricted or hindered by the boundaries of the nervous tissue. To get an idea of this relationship, we first discuss the general concept of individual spin movement and the Ensemble Average Propagator (EAP) in the presence of restricting boundaries in Section 2.1. We then discuss to a greater extent the variety and complexity of the nervous tissue in Section 2.2.

\subsection{Diffusion and the Ensemble Average Propagator}

In a fluid, water particles follow random paths according to Brownian motion [Einstein, 1956]. When we consider an ensemble of these particles in a volume, we can describe their average probability density $P(\mathbf{r} ; \tau)$ that a particle will undergo a displacement $\mathbf{r} \in \mathbb{R}^{3}$ during diffusion time $\tau \in \mathbb{R}^{+}$. This quantity is often referred to as the diffusion propagator or the ensemble average propagator (EAP) [Kärger and Heink, 1983]. In a free solution, the EAP can be described by a Gaussian distribution as

$$
P(\mathbf{r} ; \tau)=\frac{1}{\sqrt{4 \pi D \tau}} e^{-\frac{\|\mathbf{r}\|^{2}}{4 D \tau}}
$$

where $D$ is the diffusion coefficient. Eq. (1) shows that the likelihood that particles travel further increases when either $D$ or $\tau$ increases. While keeping $D$ constant, this concept can be made clear using isocontours such that $P(\mathbf{r} ; \tau)=c$ with $c>$ 0 . Figure 1 shows the same isocontour for diffusion times $\tau_{1}<\tau_{2}<\tau_{3}$ in four schematic representations of different tissue types. As can be seen by the growth of the isocontours, using longer $\tau$ increases the likelihood that particles travel further. The shape of the isocontour depends on the structure of the surrounding tissue. From left to right, in free water, where Eq. (1) is a good approximation, particles are unrestricted and travel furthest with isotropic, Gaussian probability. Next, at a course diffusion scale, gray matter tissue can often be seen as generally unorganized and hinders diffusion equally in all directions. For this reason, these tissues also produce isotropic contours, but smaller than those in free water. In axon bundles, here illustrated as gray lines, axons are mostly aligned with the bundle axis. Particle movement is restricted perpendicular to this direction and is relatively free along it, causing anisotropic isocontours [Le Bihan and Breton, 1985, Taylor and Bushell, 


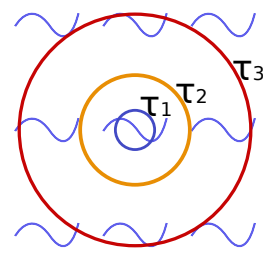

Free Water

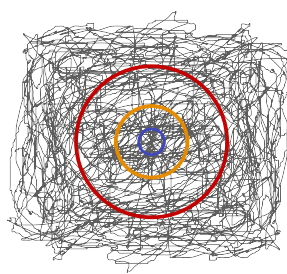

Gray Matter

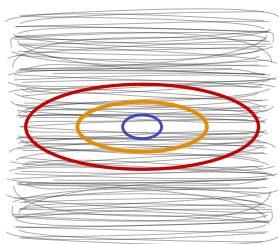

Coherent Bundle

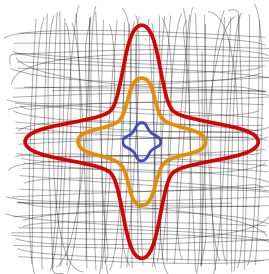

Crossing Bundles

Fig. 1: Schematic representations of different tissue types with their corresponding $P(\mathbf{r}, \tau)$ isocontours for different diffusion times $\tau_{1}<\tau_{2}<\tau_{3}$. In the "Free Water" image the blue curves just indicate the presence of water, while the grey lines in the right three images represent tissue boundaries. Longer $\tau$ lets particles travel further, indicated by the smaller blue isocontour for $\tau_{1}$ to the largest red isocontour for $\tau_{3}$. The shape of the isocontour depends on the structure of the surrounding tissue. Image inspired by Alexander [2006].

1985, Merboldt et al., 1985]. Finally, in areas where two bundles cross there is a mix between the isocontours of each bundle.

Note that we intentionally drew the isocontours for $\tau_{1}$ more isotropic than those of $\tau_{3}$ in the right two white matter tissues. For shorter $\tau$, particles have not had much time to interact with surrounding tissue, resulting in a similar probability that a particle travels in any direction. The isocontours for very short $\tau$ will therefore always be isotropic. For longer $\tau$, particles have had more time to interact with the tissue, either traveling far along a relatively unrestricted direction, or staying close to its origin along a restricted direction, resulting in more anisotropic profiles [Tanner, 1978]. When the tissue can be seen as axially symmetric (i.e. in a single bundle), this means that the perpendicular diffusivity $D_{\perp}$ becomes $\tau$-dependent and decreases as $\tau$ increases [Cohen and Assaf, 2002]. Different tissue types will induce different $\tau$-dependence of the EAP [Özarslan et al., 2006, 2012].

\subsection{Microstructure of the Brain: The Complicated Reality}

Images such as those in Fig. 1 are useful to illustrate general properties of different brain tissues. However, it is important to realize that these are extreme simplifications. Strictly speaking, the terms gray and white matter are only valid in the context of gross anatomy. Gray matter is only distinguished from white matter, in that gray matter contains numerous cell bodies and relatively few myelinated axons, while white matter is composed chiefly of long-range myelinated axon tracts and contains relatively very few cell bodies. The brain also contains glial cells of various kinds who support the functioning of neurons.

In white matter bundles, while axons are often near-tubular, their diameter, amount of myelination and the space between them varies significantly. Water par- 
ticles diffusing in different parts of this tissue, e.g. the intra- or extra-axonal space, are restricted in ways that are characteristic of that tissue type. However, the diffusion process can only be probed over large ensembles of tissues. This is illustrated by the fact that axon diameters in humans range between $0.2 \mu \mathrm{m}$ and $2 \mu \mathrm{m}$ [Aboitiz et al., 1992, Liewald et al., 2014], while the imaging resolution of diffusion MRI is around a millimeter. Appropriately dealing with the heterogeneity of the tissue and its complex influence on water diffusion is one of the big challenges of the field of diffusion MRI. In the next section, we will go into more detail on how diffusion MRI can be used to measure a signal that is related to the EAP.

\section{Measurements of Diffusion with Diffusion-Weighted MRI}

The estimation of diffusion anisotropy can be thought, in first approximation, as the assessment of the amount of preference that the diffusion process has for a specific spatial direction, compared to the others, in terms of diffusivity. Therefore, this assessment requires sensing the diffusion signal along multiple spatial directions, regardless of the representation adopted to describe the signal itself. In MRI, this is typically done by acquiring a collection of images of the target object, e.g. the brain. Each image is acquired when the experimental conditions within the magnet's bore determine a specific diffusion-weighting along the selected spatial direction: this is a Diffusion-Weighted Image (DWI). The diffusion-weighting is globally encoded by the $b$-value [Le Bihan and Breton, 1985], measured in s/ $\mathrm{mm}^{2}$, a quantity that is the reciprocal of the diffusivity, $D\left(\mathrm{~mm}^{2} / \mathrm{s}\right)$. The intensity of the diffusion-weighting, i.e. the $b$-value, is determined by the acquisition setup.

The most common type of acquisition is the Pulsed Gradient Spin-Echo sequence (PGSE) [Stejskal and Tanner, 1965], where a DWI is obtained by applying two diffusion gradients with intensity $G=\|\mathbf{G}\|(\mathrm{T} / \mathrm{m})$ and pulse length $\delta$ (s) to the tissue, separated by the separation time $\Delta$ (s). We illustrate this sequence in Figure 2. The resulting signal is 'weighted', along the applied gradient direction, with b-value [Stejskal and Tanner, 1965]

$$
b=\gamma^{2} G^{2} \delta^{2}\left(\Delta-\frac{\delta}{3}\right)
$$

where $\gamma$ is, when measuring water diffusion, the nuclear gyromagnetic ratio of the water proton ${ }^{1} \mathrm{H}$. The measurement of the diffusion signal is directly related to the concept of attenuation. Indeed, in the presence of diffusion, the signal intensities $S(b)$ of the voxels of a DWI are lower than the corresponding intensities when the image is acquired without diffusion-weighting $S_{0}=S(0)$. Along the selected gradient direction, the quantity $E(b)=S(b) / S_{0}$ expresses, for each voxel, the attenuation of the diffusion-weighted signal. In the absence of restrictions to the diffusion process, the attenuation is [Stejskal and Tanner, 1965] 


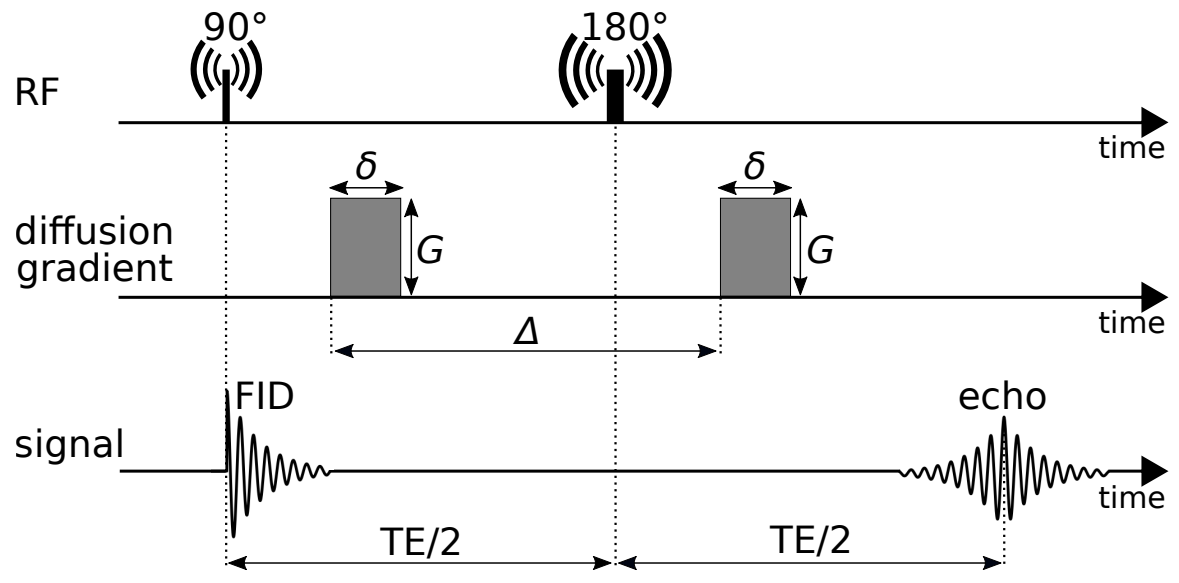

Fig. 2: Schematic illustration of the pulsed gradient spin echo sequence. The sequence is represented as the time evolution, i.e. occurrence and duration, of radiofrequency pulses (RF) in the first line, diffusion gradient pulses in the second line, and measured signal in the third line. The illustration reports the $90^{\circ}$ and $180^{\circ} \mathrm{RF}$ pulses separated by half the echo-time TE, two diffusion gradient pulses of strength $G$ and duration $\delta$ separated by a time $\Delta$, and the free induction decay (FID) and echo of the measured signal.

$$
E(b)=\frac{S(b)}{S_{0}}=e^{-b D},
$$

which expresses an exponential attenuation profile, as is illustrated in Figure $3 a$.

In the case of the PGSE sequence, this attenuation phenomenon can be interpreted as the result of a differential mechanism. The sequence, shown in Figure 2, starts with a $90^{\circ}$ radio-frequency pulse after which it is possible to measure a signal, namely Free Induction Decay (FID), that is related to the macroscopic spins' net magnetization. After a time TE/2, with TE the echo-time, a second $180^{\circ}$ radiofrequency pulse has the effect of generating an echo of the signal whose peak is at time TE, corresponding to the end of the sequence [Hahn, 1950]. The first diffusion gradient pulse is applied between the two radio-frequency pulses. Here, we assume the narrow gradient pulse condition $\delta \ll \Delta$, which implies that spins are static during the application of the gradient pulses [Tanner and Stejskal, 1968]. Under this assumption, after the first gradient pulse, a spin located at position $\mathbf{r}_{1}$ is subject to a phase accumulation $\phi_{1}=\gamma \delta \mathbf{G} \cdot \mathbf{r}_{1}$. After a time $\Delta$ from the start of the first gradient pulse, and after the $180^{\circ}$ radio-frequency pulse, a second gradient pulse of equal magnitude and duration to the first is applied. If the spin has moved to a position $\mathbf{r}_{2}$ the phase accumulation during the second pulse is $\phi_{2}=\gamma \delta \mathbf{G} \cdot \mathbf{r}_{2}$. However, the $180^{\circ}$ radio-frequency pulse has the effect of changing the sign of the second gradient pulse. Therefore, at the end of the sequence, i.e. at the echo-time TE, the spin has acquired a net phase shift 


$$
\phi=\phi_{2}-\phi_{1}=\gamma \delta \mathbf{G} \cdot\left(\mathbf{r}_{2}-\mathbf{r}_{1}\right)=\gamma \delta \mathbf{G} \cdot \mathbf{r}
$$

which is null in the case the spin remained static, i.e. $\mathbf{r}=\mathbf{r}_{2}-\mathbf{r}_{1}=0$.

The signal attenuation takes into account an ensemble of spins and can be related to the ensemble average propagator (EAP), $P(\mathbf{r}, \tau)$, via a Fourier relationship under the q-space formalism [Tanner and Stejskal, 1968, Callaghan, 1991]

$$
E(\mathbf{q}, \tau)=\int_{\mathbb{R}^{3}} P(\mathbf{r}, \tau) e^{j 2 \pi \mathbf{q} \cdot \mathbf{r}} d \mathbf{r}
$$

where $\mathbf{q}$ is the wave vector and $\tau$ is diffusion time, which for the PGSE sequence are expressed as

$$
\mathbf{q}=\frac{\gamma \delta \mathbf{G}}{2 \pi} \text { and } \tau=\Delta-\delta / 3 .
$$

These quantities influence differently the diffusion-weighting, i.e. the b-value. Indeed, the wave frequency $q=\|\mathbf{q}\|$ is expressed in $\mathrm{mm}^{-1}$ and is the reciprocal of the spin distance $r=\|\mathbf{r}\|$ expressed in $\mathrm{mm}$. As such, by increasing the measured spatial frequency $q$, we can obtain a higher resolution of its inverse Fourier transform, the diffusion propagator $P(\mathbf{r}, \tau)$ described by $r$. In addition, the diffusion time $\tau$ expresses the time interval during which spins are allowed to diffuse before measurement. A longer diffusion time allows the spins to move a longer distance causing, in the absence of restrictions to the diffusion process, a larger net phase shift, i.e. a stronger attenuation of the signal. Therefore, expressing the diffusion-weighting in terms of $\mathbf{q}$ and $\tau$ can provide useful insights on the signal nature. In the absence of restrictions to the diffusion process, eq. (5) has a closed form. This is obtained by substituting $q$ and $\tau$ of eq. (6) into the formulation of the b-value expressed in eq. (2), such that $b=4 \pi^{2} q^{2} \tau$ and eq. (3) becomes

$$
E(q, \tau)=e^{-4 \pi^{2} q^{2} \tau D}
$$

which expresses a Gaussian attenuation profile as function of $q$. However, eqs. (3) and (7) are valid when the diffusion process can be considered unrestricted, e.g. when the movement of spins is not obstructed by the presence of a barrier. In the case of restricted diffusion, for instance when the signal is measured along a direction perpendicular to a barrier, these equations are no longer valid.

Unrestricted and restricted scenarios are depicted by the schematic representation in the left side of Figure 3, where the diffusion process occurs between two parallel barriers, i.e. the restriction, and where two arrows represent the measurement directions parallel and perpendicular to the restriction. The figure also illustrates the signal attenuation in the case of parallel, unrestricted diffusion $(a, b)$, and in the case of perpendicular, restricted diffusion $(c, d)$. The unrestricted attenuations are obtained with eqs. (3) and (7), whereas the restricted ones are simulated as the diffusion signal attenuation generated within an ensemble of cylinders along the direction perpendicular to the cylinders' axes [Callaghan, 1995]. Moreover, the curves in $a$ and $c$ are reported as function of the b-value with diffusion time $\tau_{2}$, whereas the curves in $b$ and $d$ are functions of the q-value and are reported for increasing 

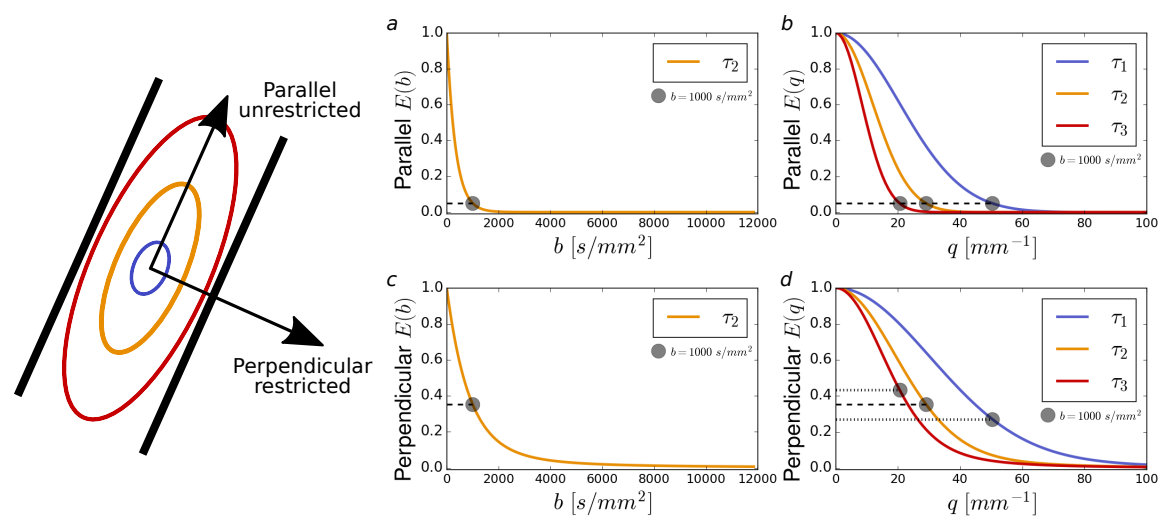

Fig. 3: The effect of b-value, q-value and diffusion time on the signal attenuation when diffusion is unrestricted or restricted by the presence of a barrier (a cylinder). The graphs report the signal attenuations along a direction parallel to the restriction $(a, b)$ - represented by two black barriers in the schematic image - and along the perpendicular direction $(c, d)$, where the diffusion process is restricted. The attenuations are reported as function of the b-value $(a, c)$ with diffusion time $\tau_{2}$, and q-value $(b, d)$ for increasing diffusion times $\tau_{1}<\tau_{2}<\tau_{3}$. Dots indicate the attenuation measured at $b=1000 \mathrm{~s} / \mathrm{mm}^{2}$. Note that if we were to plot the single cylinder signal attenuation in $d$ in log-scale we would find diffraction patterns [Callaghan, 1995], but in practice these are never visible because nervous tissues contain distributions of axon diameters, whose diffraction patterns average out to a smooth line.

diffusion times $\tau_{1}<\tau_{2}<\tau_{3}$. In the graphs, points of each curve corresponding to $b=1000 \mathrm{~s} / \mathrm{mm}^{2}$ are highlighted with a dot.

Along the unrestricted direction $(a, b)$ the attenuation values, indicated by dots, are lower than the corresponding ones along the restricted direction $(c, d)$. Indeed, when diffusion is restricted by the presence of the barrier, the spins are subject to a smaller net displacement and the signal attenuates less.

The choice of $q$ and $\tau$ to obtain a certain diffusion-weighting, i.e. a specific b-value, assumes different relevance in terms of signal attenuation depending on whether diffusion is restricted or not. In the absence of restrictions $(a, b)$, an increase of q-value or diffusion time always attenuates the signal, and points with different $q$ and $\tau$, but with same b-value, render the same amount of attenuation $(b)$. However, when diffusion is restricted $(c, d)$, an increase of the diffusion time $\tau$ implies letting the spins diffuse a longer distance with the consequence of experiencing more restriction. In this case, the Gaussian attenuation expressed by eq. (7) is not longer valid. Indeed, different combinations of $q$ and $\tau$ render different non-Gaussian profiles of signal attenuation, and points with same b-value - the dots of Figure $3 d-$ correspond to different attenuations.

The estimation of diffusion anisotropy, based on the diffusion signal attenuation along different gradient directions, depends on the chosen experimental pa- 


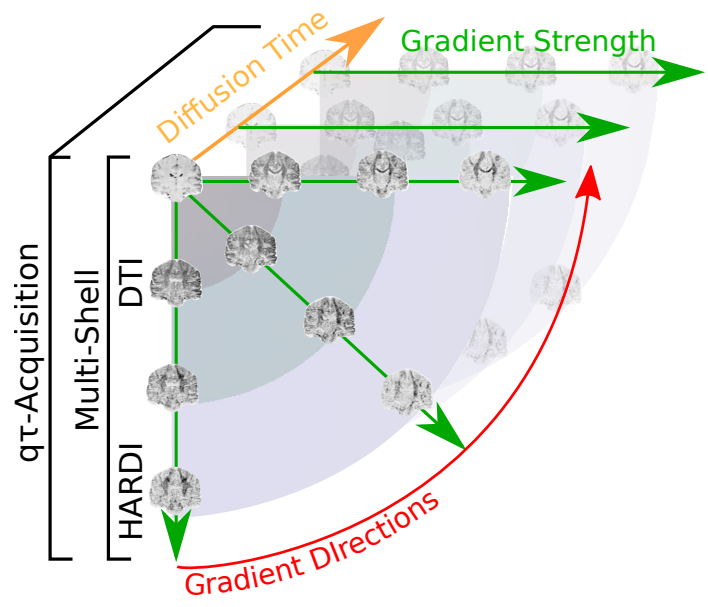

Fig. 4: Schematic for different types of acquisition schemes. DTI schemes are typically acquired at a low gradient strength, typically $b<1000 \mathrm{~s} / \mathrm{mm}^{2}$, for a minimum of 6 gradient directions. HARDI typically uses higher gradient strengths, typically $b>2000 \mathrm{~s} / \mathrm{mm}^{2}$ with over 40 gradient directions. Multi-shell uses DTI and HARDI shells for a given diffusion time, and finally q $\tau$-acquisitions are basically multi-shell acquisitions for a range of diffusion times.

rameters, especially $q$ and $\tau$. Indeed, different sets of parameters lead to different signal attenuations - depending on the underlying diffusion process - and consequently to different measurements of anisotropy. Ideally, a complete characterization of anisotropy would require the measurement of the diffusion signal attenuation for many gradient directions, q-values, and diffusion times $\tau$. However the optimal sampling is still under debate. In practice, with reference to diffusion anisotropy, the choice of how to sample the diffusion signal depends on the application and on the chosen signal representation. For instance, in DTI it is common practice to measure only one sample, i.e. one b-value, per gradient direction [Le Bihan et al., 2001] as illustrated in Figure 4. However, with High Angular Resolution Diffusion Imaging (HARDI) the number of directions - typically above 40 - and the b-value is considerably increased to obtain a signal representation with a higher angular resolution [Tuch et al., 2002]. Other signal representations require signal acquisition at different diffusion-weightings. A common choice, namely multi-shell, consists in acquiring different $\mathrm{q}$-shells while fixing the diffusion time. Each shell represents a collection of samples in the three-dimensional space with the same q-value. These samples can be imagined as lying on a sphere, as shown in Figure 4, and it is convenient to distribute them uniformly on the spherical surface to obtain an optimal spatial coverage. This concept can be expanded among shells such that all of the acquired samples lie on different non-collinear directions [Caruyer et al., 2013]. The multi-shell concept can be extended to $\tau$-shells, called a $\mathrm{q} \tau$-acquisition [Fick et al., 2016b], since nowadays there exist signal representations that exploit different value 
for both $q$ and $\tau$. In this case, a complete q-shell scheme - with samples distributed along different gradient directions and with different diffusion-weightings - is acquired for each desired diffusion time.

\section{The Inter-Model Variability of Diffusion Anisotropy}

We now return to the metric that is most commonly used as a marker for changes in tissue microstructure: diffusion anisotropy. Simply meaning "deviation from diffusion isotropy", different interpretations of diffusion anisotropy have been proposed using different acquisition requirements and mathematical underpinnings [Basser, 1995, Tuch, 2004, Özarslan et al., 2013, Kaden et al., 2015]. Out of these, the clinical applications of Fractional Anisotropy (FA) [Basser, 1995] of the Diffusion Tensor Imaging (DTI) model [Basser et al., 1994] has been most widely explored. Changes in FA have been related to brain diseases such as ischemia, multiple sclerosis, trauma, brain tumors and many more [see e.g. reviews by Assaf and Pasternak, 2008, Soares et al., 2013]. For this reason, FA is seen as a "potential biomarker" for these disease patterns, where biomarker is a portmanteau of "biological marker" [Strimbu and Tavel, 2010]. However, the fact that FA is sensitive to all these processes also means that it is specific to none of them.

Diffusion anisotropy measures, as a rule of thumb, always have the following three properties:

- They are rotationally invariant, i.e., insensitive to rotations.

- They are normalized, with zero being the lowest measure for diffusion anisotropy and one being the highest.

- They somehow describe "deviation from diffusion isotropy".

The last point is intentionally left open to interpretation, which is exactly the point we are making in this section. To illustrate this, we discuss seven different anisotropy measures; Fraction Anisotropy (FA) [Basser, 1995], Relative Anisotropy (RA), Kurtosis Fractional Anisotropy (KFA), Generalized Fraction Anisotropy (GFA) [Tuch, 2004]; Propagator Anisotropy (PA) [Özarslan et al., 2013]; Orientation Dispersion Index (ODI) [Zhang et al., 2012]; and microscopic Fractional Anisotropy ( $\mu$ FA) [Kaden et al., 2015]. All of these except ODI and $\mu$ FA are signal-based metrics for diffusion anisotropy. We added these two to illustrate that the concept of diffusion anisotropy transcends signal-based metrics.

We start this section by first detailing the data of the Human Connectome Project [Setsompop et al., 2013] that we use to illustrate different diffusion anisotropy measures in Section 4.1. We then describe the inter-model variability of the mathematical definition and estimation of signal-based diffusion anisotropy in Section 4.2. We then detail anisotropy as a property of axon dispersion of micro-environments (ODI) in Section 4.3, and as a property of one micro-environment ( $\mu \mathrm{FA})$ in Section 4.4. The anisotropy measures of all presented techniques are qualitatively and quantitative compared in Fig. 5, 6 and 7. 


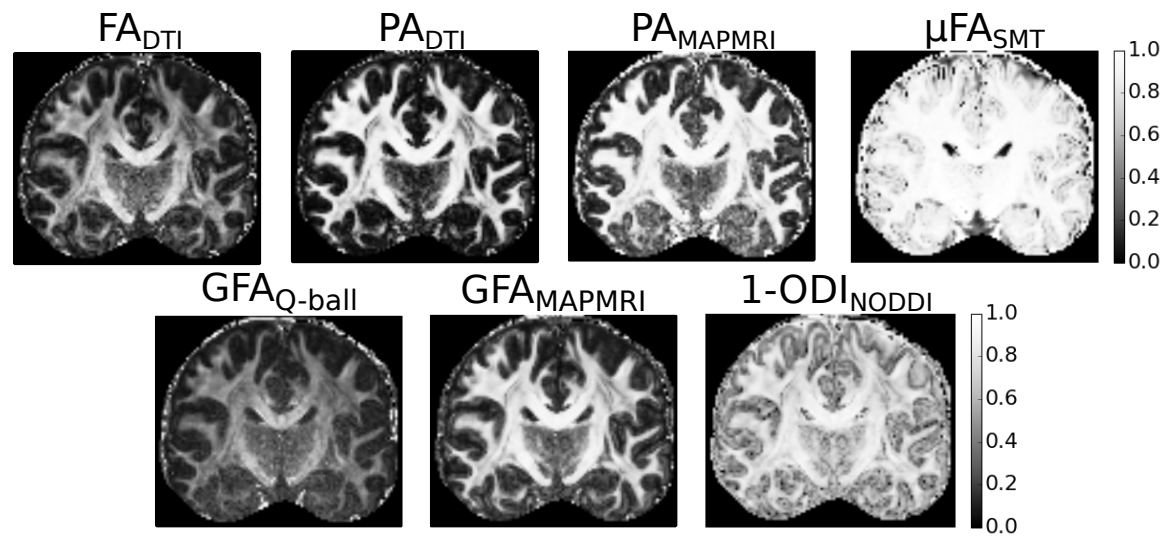

Fig. 5: Visualization of various normalized measures describing diffusion anisotropy, where the corresponding model is given in the subscript. In the top row, we show Fractional Anisotropy (FA) and Propagator Anisotropy (PA) of Diffusion Tensor Imaging (DTI), PA by Mean Apparent Propagator (MAP)-MRI and microFA by Spherical Mean Technique (SMT). In the bottom row, we show Generalized Fractional Anisotropy (GFA) by Q-ball Imaging and by MAP-MRI and finally one minus the Orientation Dispersion Index (ODI) by Neurite Orientation Dispersion and Density Imaging (NODDI). The complement of ODI is shown for overall coherence, since high ODI normally indicates low anisotropy.

\subsection{Data Set Description and Adopted Notation}

We use the MGH Adult Diffusion Data of the Human Connectome Project to illustrate different measures of diffusion anisotorpy [Greve and Fischl, 2009, Andersson et al., 2012, Keil et al., 2013, Setsompop et al., 2013]. This data set was acquired at particularly high b-values $\{0,1000,3000,5000,10000\} \mathrm{s} / \mathrm{mm}^{2}$ with $\{40$, $64,64,128,256\}$ directions, respectively. The diffusion time and pulse separation time in this data are $\delta / \Delta=12.9 / 21.8 \mathrm{~ms}$ with $1.5 \times 1.5 \times 1.5 \mathrm{~mm}$ resolution and $T E / T R=57 / 8800 \mathrm{~ms}$.

\subsection{Diffusion Anisotropy as a Signal Property}

In this section, we discuss five different anisotropy measures known as Fraction Anisotropy (FA) [Basser, 1995], Relative Anisotropy (RA), Kurtosis Fractional Anisotropy (KFA) [Jensen et al., 2005], Generalized Fraction Anisotropy (GFA) [Tuch, 2004] and Propagator Anisotropy (PA) [Özarslan et al., 2013].

Signal-based models directly estimate the EAP $P(\mathbf{r} \mid \tau)$ from the measured signal attenuation $E(\mathbf{q}, \tau)$, using the Fourier relationship in Eq. (5). Notice that $P(\mathbf{r} \mid \tau)$ is a 

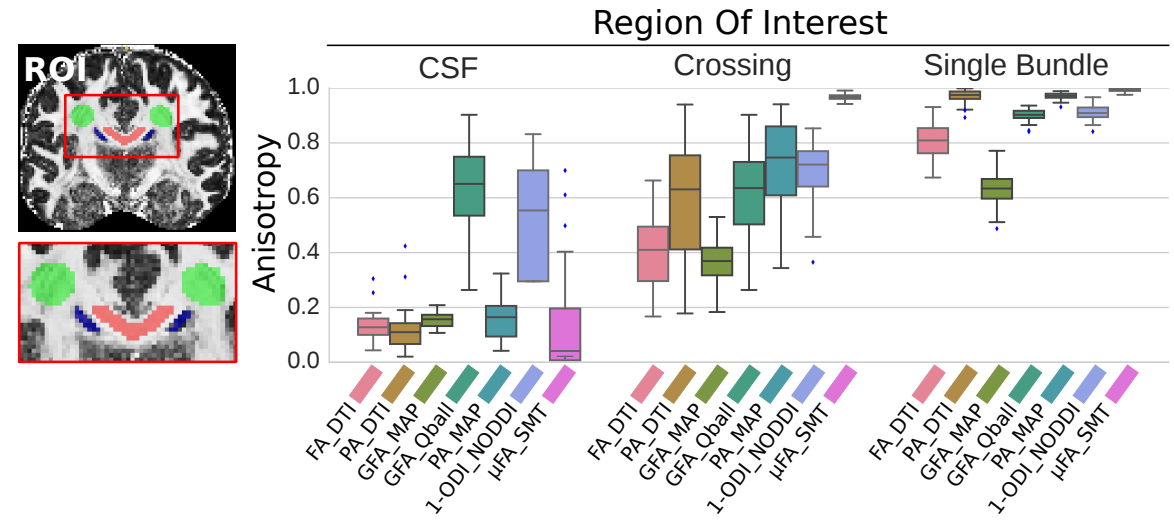

Fig. 6: Box-plots of anisotropy measures in estimated in different regions of interest, indicated as the different colors in the coronal brain slice in the right corner. We illustrate anisotropy in free water (CSF), a single bundle (Corpus Callosum) and a crossing area (Centrum Semiovale). It can be seen that the same metric for different techniques, or different metrics for the same technique can result in different estimates of anisotropy. Note that the estimates of $\mu \mathrm{FA}$ (the most-right metric per group in pink) in the crossing and single bundles are so consistent that the boxplots appear almost flat.

conditional probability density for diffusion time $\tau$, as the Fourier transform is only over the $\mathbf{q}, \mathbf{r}$ space. We will interchangeably use real displacement vector $\mathbf{r}=r \mathbf{u}$ with its distance and direction $r \in \mathbb{R}^{+}, \mathbf{u} \in \mathbb{S}^{2}$ and q-space vector $\mathbf{q}=q \mathbf{g}$ with its $\mathrm{q}$-space distance and gradient orientation $q \in \mathbb{R}^{+}, \mathbf{g} \in \mathbb{S}^{2}$. The following anisotropy measures are then defined as some difference or ratio between the isotropic and anisotropic parts of $P(\mathbf{r} \mid \tau)$.

Relative and Fractional Anisotropy Starting with one of the oldest measures for diffusion anisotropy, Relative Anisotropy (RA) and Fractional Anisotropy (FA) [Basser, 1995] are specific for the DTI model [Basser et al., 1994]. DTI solves the Fourier transform by generalizing the Stejskal-Tanner equation for unbounded media [Tanner and Stejskal, 1968] to three dimensions:

$$
E(b)=\exp \left(-b \mathbf{g}^{\mathrm{T}} \mathbf{D g}\right) \quad \text { or } \quad E(\mathbf{q}, \tau)=\exp \left(-4 \pi^{2} q^{2} \tau \mathbf{g}^{\mathrm{T}} \mathbf{D g}\right)
$$

with $\mathbf{D}$ a $3 \times 3$ symmetric positive-definite diffusion tensor. Notice that Eq. (8) is Gaussian over $q$ and exponential over $\tau$, which will be important in studying timedependence in Section 5. FA describes fraction of the "magnitude" of $\mathbf{D}$ that we can ascribe to anisotropic diffusion in terms of its eigenvalues $\left\{\lambda_{1}, \lambda_{2}, \lambda_{3}\right\}$, wheres RA divides the magnitude of the anisotropic part by that of the isotropic part as 


$$
\begin{aligned}
& F A=\frac{\operatorname{std}(\lambda)}{\operatorname{rms}(\lambda)}=\sqrt{\frac{1}{2}} \frac{\sqrt{\left(\lambda_{1}-\lambda_{2}\right)^{2}+\left(\lambda_{2}-\lambda_{3}\right)^{2}+\left(\lambda_{3}-\lambda_{1}\right)^{2}}}{\sqrt{\lambda_{1}^{2}+\lambda_{2}^{2}+\lambda_{3}^{2}}} \\
& R A=\frac{\operatorname{std}(\lambda)}{\operatorname{mean}(\lambda)}=\frac{3 \sqrt{\left(\lambda_{1}-\lambda_{2}\right)^{2}+\left(\lambda_{2}-\lambda_{3}\right)^{2}+\left(\lambda_{3}-\lambda_{1}\right)^{2}}}{\lambda_{1}+\lambda_{2}+\lambda_{3}} .
\end{aligned}
$$

Both measures are zero when the medium is isotropic, but only FA is normalized between zero and one, which likely led to the prevalence of FA over RA in the community. The interpretation of FA has known limitations as DTI cannot represent crossing tissue configurations, but finds some average, Gaussian approximation that best fits the overall signal [Basser et al., 1994]. We illustrate this by estimating DTI's Orientation Distribution Function $\operatorname{ODF}(\mathbf{u} \mid \tau)$, representing the probability density that a diffusing particle will travel along direction $\mathbf{u}$ by marginalizing $r$ as

$$
O D F(\mathbf{u} \mid \tau)=\int_{0}^{\infty} P(r \mathbf{u} \mid \tau) r^{2} d r
$$

where $r^{2}$ is the Jacobian of the radial integration to ensure that the integral of the ODF is unity [Tristán-Vega et al., 2009, Aganj et al., 2010]. Notice that Eq. (11) is general, and can be used for any method that estimates $P(r \mathbf{u} \mid \tau)$. For instance, Eq. (11) can be given analytically for DTI as

$$
O D F_{\mathrm{DTI}}(\mathbf{u})=\frac{1}{4 \pi|\mathbf{D}|^{\frac{1}{2}}\left(\mathbf{u}^{\mathrm{T}} \mathbf{D}^{-1} \mathbf{u}\right)^{\frac{3}{2}}}
$$

We show DTI's ODFs in a crossing area in Fig. 7, where it can be seen that round profiles with low FA are found where other methods detect crossings.

Kurtosis Fractional Anisotropy In Diffusion Kurtosis Imaging (DKI) [Jensen et al., 2005] the non-Gaussian aspects of the signal attenuation are represented using the Taylor expansion. Using a summation instead a matrix product like in Eq. (8), DKI describes the signal attenuation as

$$
E(b)=\exp \left(-b \sum_{i, j}^{3} g_{i} g_{j} D_{i j}+\frac{b^{2}}{6} \bar{D}^{2} \sum_{i, j, k, l}^{3} g_{i} g_{j} g_{k} g_{l} W_{i j k l}+O\left(b^{3}\right)\right)
$$

where $\bar{D}$ is the mean diffusivity with Diffusion Tensor $\mathbf{D}$ and non-Gaussian Kurtosis Tensor W. In complete analogy to FA, the Kurtosis Fractional anisotropy is defined as

$$
K F A=\frac{\operatorname{std}(\mathbf{W})}{\operatorname{rms}(\mathbf{W})} .
$$

KFA therefore represents the anisotropy of the non-Gaussian aspects of the signal. DKI-based metrics have been shown to be more sensitive to pathology that DTI-based ones [Cheung et al., 2009] and variations of its definition in terms of directional variation have been explored [Hansen and Jespersen, 2016]. 
ODFs / FODs in Crossing Area for Different Methods

DTI ODFs with FA Background

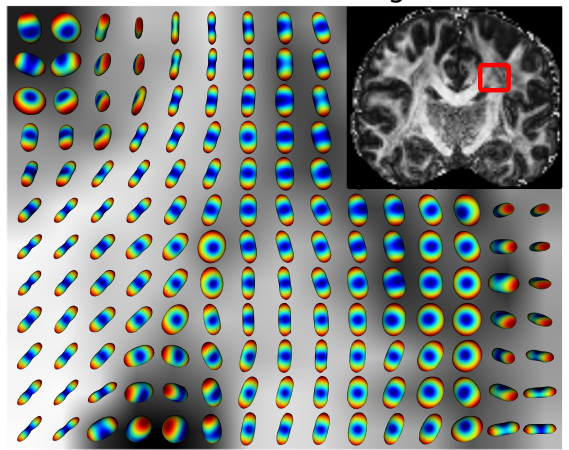

MAPMRI ODFs with PA Background

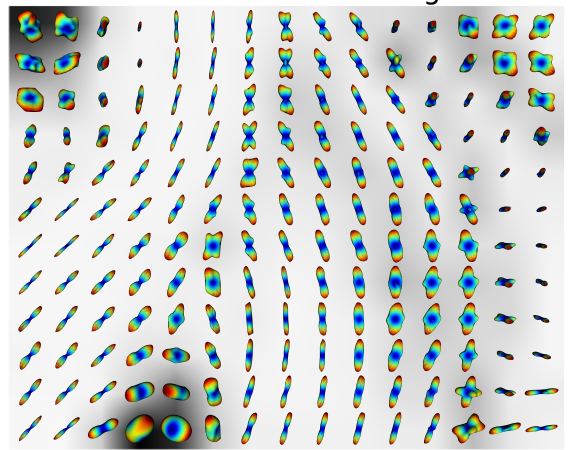

Q-ball ODFs with GFA Background

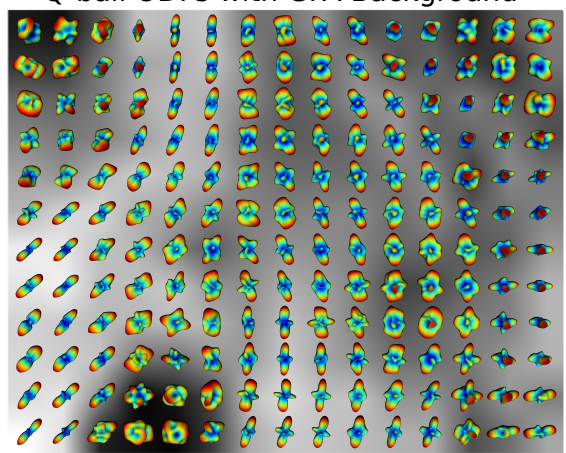

SMT FODs with $\mu$ FA Background

* $8188888: \times x \times$ $+x+188 x+18 x+8$

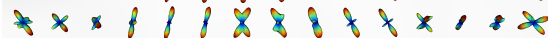

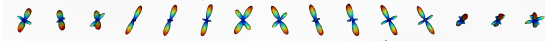
$\& 8 \& 8$ \& $f \times x+f+t+\ldots$ $888 \times 8 \times \& \&$ if $t+t$. $88 \times 8+x \times t+i f+t \ldots$

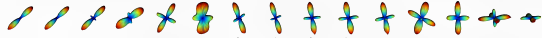

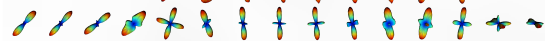
$888+x+i$ if $f$ if $f=\cdots$

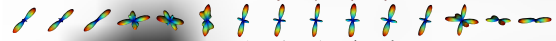
$8<x \times$ of $f$ if $f f-\infty$ Watson ODFs with 1-ODI Background

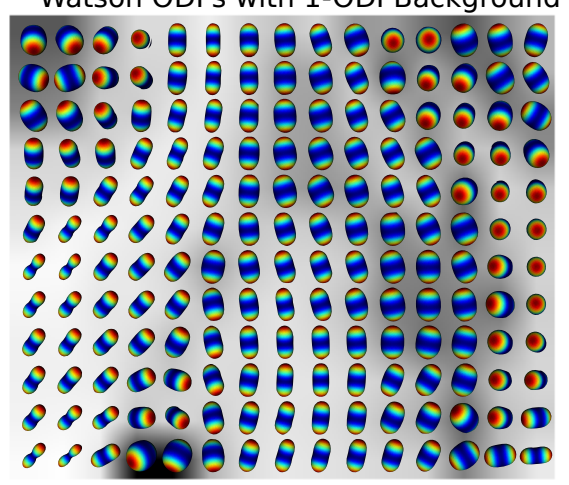

Fig. 7: Orientation Distribution Functions (ODFs) for DTI, Q-ball, MAPMRI, the Watson distributions of NODDI and Fiber Orientation Distributions (FODs) of SMT in an area where it is known there are crossing bundles. Each method has its corresponding anisotropy measure as background texture. It can be seen that DTI finds an average orientation, where Q-Ball, MAPMRI and SMT find crossing structures. The FODs, being the result of a deconvolution, show sharper peaks, and more consistent crossings than the ODF techniques. It is important to realize that while NODDI separates the signal contributions of intra- and extra-axonal diffusion, its dispersion index jointly describes the spread of both of these compartments, and produces very similar ODFs as DTI. 
Generalized Fractional Anisotropy GFA [Tuch, 2004] was proposed for High Angular Resolution Diffusion Imaging (HARDI) techniques [Tuch et al., 2002], that use the Funk-Radon Transform (FRT) to estimate ODFs capable of describing multiple axon directions [Tuch et al., 2003]. It is noteworthy that the only difference between DTI and HARDI is that in HARDI more gradients $\mathbf{g}$ are measured at a higher b-values to gain a better angular resolution of the ODF, see Fig. 4. This means that still no radial information is known of $P(r \mathbf{u} \mid \tau)$, and Gaussian decay over $r$ is assumed to estimate the ODF in Eq. (11). For any ODF, the GFA is given as

$$
G F A=\frac{\operatorname{std}(O D F)}{\operatorname{rms}(O D F)}=\sqrt{\frac{n \sum_{i=1}^{n}\left(O D F\left(\mathbf{u}_{i}\right)-\langle O D F(\mathbf{u})\rangle\right)^{2}}{(n-1) \sum_{i=1}^{n} O D F\left(\mathbf{u}_{i}\right)^{2}}}
$$

where $\operatorname{ODF}(\mathbf{u})$ is the value of the ODF in direction $\mathbf{u}, n$ is the number of evaluated ODF directions and $\langle O D F(\mathbf{u})\rangle$ is the mean ODF intensity. We show Q-ball Imaging ODFs [Tuch, 2004, Descoteaux et al., 2007, Aganj et al., 2010] in the top-right of Fig. 7, where now crossing structures can be seen. Though, it must be said that FRT has been applied to a variety of HARDI methodologies, a selection of which is summarized in Tristan-Vega et al. [2010]. Moreover, notice that GFA intensities in the crossing and single bundles areas are more similar than seen with DTI's FA in the top-left, but the overall intensities of GFA and FA are different. We illustrate this by comparing estimated anisotropy intensities in different ROIs, for different measures and techniques in Fig. 6. It can be seen that the same metric for different techniques, or different metrics for the same technique can result in different estimates of anisotropy.

Propagator Anisotropy PA was proposed for the multi-shell, Mean Apparent Propagator (MAP)-MRI technique [Özarslan et al., 2013]. MAP-MRI can be seen as a generalization of DTI, and allows for the estimation of three-dimensional $P(r \mathbf{u} \mid \tau)$, where now both restricted (non-Gaussian) diffusion over $r$ and crossing axons can be represented. MAP-MRI is not unique in this respect, as a plethora of multi-shell techniques have been proposed for this purpose [See e.g. Assemlal et al., 2009, Descoteaux et al., 2011, Hosseinbor et al., 2013, Rathi et al., 2014], but MAP-MRI's formulation allows for easy estimation of a large variety of q-space properties using efficient regularization [Fick et al., 2016c]. PA is defined as a measure of dissimilarity between the reconstructed $P(\mathbf{r} \mid \tau)$ and its closest isotropic approximation $P_{\text {iso }}(\mathbf{r} \mid \tau)$. First, the inner product between two EAPs is defined as

$$
\left\langle P(\mathbf{r} \mid \tau) P_{\text {iso }}(\mathbf{r} \mid \tau)\right\rangle=\int_{\mathbb{R}^{3}} P(\mathbf{r} \mid \tau) P_{\text {iso }}(\mathbf{r} \mid \tau) d \mathbf{r} .
$$

The similarity between two propagators is measured as an angular measure of covariance in analogy with the vector product [Avram et al., 2015]:

$$
\cos \theta_{\mathrm{PA}}=\sqrt{\frac{\left\langle P(\mathbf{r} \mid \tau) P_{\mathrm{iso}}(\mathbf{r} \mid \tau)\right\rangle}{\langle P(\mathbf{r} \mid \tau) P(\mathbf{r} \mid \tau)\rangle\left\langle P_{\mathrm{iso}}(\mathbf{r} \mid \tau) P_{\mathrm{iso}}(\mathbf{r} \mid \tau)\right\rangle}}
$$


$\mathrm{PA}$ is then defined using the angular dissimilarity measure $\sin \theta_{\mathrm{PA}}=\sqrt{1-\cos \theta_{\mathrm{PA}}^{2}}$ and scaling function $\sigma(t, \varepsilon)$ as

$$
P A=\sigma\left(\sin \theta_{\mathrm{PA}}, 0.4\right) \quad \text { with } \quad \sigma(t, \varepsilon)=\frac{t^{3 \varepsilon}}{1-3 t^{\varepsilon}+3 t^{2 \varepsilon}} .
$$

where we note that scaling parameter $\varepsilon=0.4$ was chosen by Özarslan et al. [2013] to yield "the desired level of contrast in real images". PA can be estimated for any method that reconstructs $P(\mathbf{r} \mid \tau)$. For instance, for DTI it is given as

$$
\cos \theta_{\text {PA-DTI }}^{2}=\frac{8 u_{0}^{3} u_{x} u_{y} u_{z}}{\left(u_{x}^{2}+u_{0}^{2}\right)\left(u_{y}^{2}+u_{0}^{2}\right)\left(u_{z}^{2}+u_{0}^{2}\right)}
$$

where the displacement is given as a function of DTI's eigenvalues as $\left\{u_{x}, u_{y}, u_{z}\right\}=$ $\sqrt{2 \tau\left\{\lambda_{1}, \lambda_{2}, \lambda_{3}\right\}}$ [Basser, 2002], and $u_{0}$ is DTI's nearest isotropic propagator [Özarslan et al., 2013]. We show $P A_{\mathrm{MAPMRI}}$ and $P A_{\mathrm{DTI}}$ in Fig. 5 and 6, where this measure indeed seems to show good contrast between isotropic, crossing and single bundle tissues.

\subsection{Anisotropy as Orientation Dispersion of Micro-Environments}

As the only multi-compartment model that we consider in this chapter, the neurite orientation dispersion and density imaging (NODDI) model [Zhang et al., 2012] parameterizes diffusion anisotropy as the dispersion of the diffusion signal of individual axon segments around a central bundle axis. In NODDI, axons as are represented as sticks - cylinders with zero radius and parallel diffusivity $\lambda_{\|}$- and are dispersed according to a Watson distribution $W(\kappa, \boldsymbol{\mu})$, where $\kappa$ is the concentration parameter that is inversely related to axon dispersion, and $\boldsymbol{\mu} \in \mathbb{S}^{2}$ is the bundle direction. NODDI also separates the signal contribution of the Cerebrospinal Fluid (CSF) as an isotropic Gaussian with diffusivity $D_{\text {iso }}$ (i.e. a Ball) and the hindered hindered extra-axonal compartment - the diffusion directly around the axons - as an axially symmetric Tensor (i.e. a Zeppelin) with parallel and perpendicular diffusivity $\lambda_{\|}^{\text {ext }}$ and $\lambda_{\perp}^{\text {ext }}$. The overall signal representation is then

$$
E_{\text {Watson }}^{\mathrm{NODDI}}=\underbrace{f_{\mathrm{CSF}} \overbrace{E_{\mathrm{iso}}\left(D_{\mathrm{CSF}}\right)}^{\text {Ball }}}_{\text {CSF }}+\overbrace{W(\kappa, \boldsymbol{\mu})}^{\text {Watson }} * *_{\mathbb{S}^{2}}[\underbrace{f_{h} \overbrace{E_{\mathrm{h}}\left(\lambda_{\perp}^{\text {ext }}, \lambda_{\|}^{\text {ext }}\right)}^{\text {Zeppelin }}}_{\text {Hindered Extra-Axonal }}+\underbrace{f_{r} \overbrace{E_{r}\left(\lambda_{\|}\right)}^{\text {Stick }}}_{\text {Intra-Axonal }}]
$$

where the volume fractions of the CSF, hindered and intra-axonal compartment sum up to unity as $f_{\mathrm{CSF}}+f_{h}+f_{r}=1$, and $*_{\mathbb{S}^{2}}$ represents the spherical convolution that distributes the per-axon diffusion signal (both the stick and the hindered compartment) according to the Watson distribution [Kaden et al., 2007]. In prac- 
tice, NODDI fixes all diffusivities of the different model components and focuses on the estimation of $\kappa, \boldsymbol{\mu}$ and the volume fractions, leading to biases when the preset diffusivities don't reflect the true diffusivities. On the other hand, not fixing any diffusivities and simultaneously fitting all parameters leads to multi-modallity in the parameters space - different tissue representations can produce the same diffusion signal [Jelescu et al., 2016].

NODDI reparameterizes the concentration parameter $\kappa$ into the normalized Orientation Dispersion Index (ODI)

$$
\text { ODI }=\frac{2}{\pi} \arctan (1 / \kappa)
$$

where ODI $=0$ now means no dispersion, i.e. parallel axons and therefore high diffusion anisotropy, and ODI $=1$ represents completely dispersed (isotropic) diffusion. We fitted the NODDI model using the NODDI toolbox ${ }^{1}$ and illustrate the complement of ODI in Figs. 5 and 6. It can be seen that the contrast of previously discussed signal-based anisotropy measures and ODI is quite similar. Fig. 7 also shows the ODFs of the estimated Watson distributed, which resemble the DTI ODFs in many cases.

\subsection{Anisotropy as a Property of Micro-Environments}

Similarly as NODDI, the Spherical Mean Technique (SMT) [Kaden et al., 2015] also represents the diffusion signal as a distribution of individual axon segments with a spherical Fiber Orientation Distribution $\operatorname{FOD}(\mathbf{n})$. However, unlike NODDI, SMT does not do any separately model the hindered and CSF compartments, nor does it assume any parameterization of the FOD, and only assumes that the FOD is a probability density such that $\int_{\mathbb{S}^{2}} \operatorname{FOD}(\mathbf{n})=1$ and the individual axon segment is represented by an axially symmetric tensor with perpendicular and parallel diffusivity $\lambda_{\perp}$ and $\lambda_{\|}$. It then follows that, for a given b-value, the spherical integral $\varepsilon$ of the overall diffusion signal $E_{b}(\mathbf{n})$ and that of the individual axon segment $K_{b}(\mathbf{n})$ must be equal such that

$$
\varepsilon_{E}(b)=\int_{\mathbb{S}^{2}} E_{b}(\mathbf{n}) d \mathbf{n}=\int_{\mathbb{S}^{2}}\left(\mathrm{FOD} *_{\mathbb{S}^{2}} K_{b}\right)(\mathbf{n}) d \mathbf{n}=\int_{\mathbb{S}^{2}} K_{b}(\mathbf{n}) d \mathbf{n}=\varepsilon_{K}\left(b, \lambda_{\perp}, \lambda_{\|}\right) .
$$

It is possible to solve this equation for $\lambda_{\perp}$ and $\lambda_{\|}$using constrained least squares such that $0<\lambda_{\perp}<\lambda_{\|}<\lambda_{\text {free }}$ with $\lambda_{\text {free }}$ the free water diffusivity. Once $\lambda_{\|}, \lambda_{\perp}$ are known, the per-axon fractional anisotropy ( $\mu \mathrm{FA})$ is calculated as in Eq. (9), with $\lambda_{1}=\lambda_{\|}$and $\lambda_{2}=\lambda_{3}=\lambda_{\perp}$. As we show in Figs. 5 and 6, the estimation of $\mu \mathrm{FA}$ in the SMT framework is now independent of axon dispersion or crossing tissue configurations, that are very noticeable in for instance FA and ODI. However, SMT cannot distinguish between axon bundles, meaning that when two bundles

${ }^{1}$ http://www.nitrc.org/projects/noddi_toolbox 


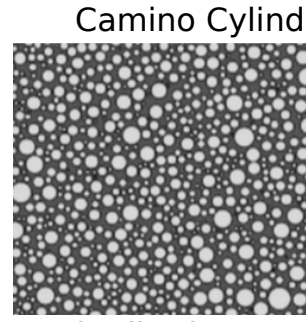

Distribution 1

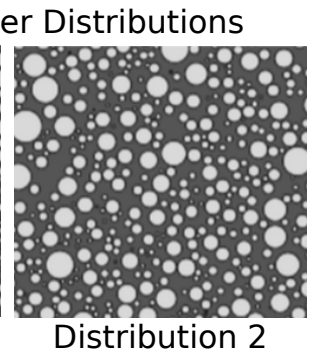

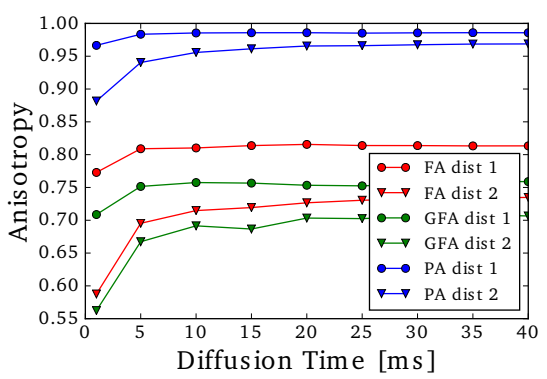

Fig. 8: left: Cross-sections of Camino cylinder substrates with gamma-distributed radii. Distribution 1 has smaller radii and less extra-cellular space than distribution 2. right: Diffusion time dependence of anisotropy measures from the two left distributions. The estimated anisotropy increases as longer diffusion times are used, until a plateau is reached.

with different diffusivities exist within one voxel the method can only estimate the average of the two. Lastly, the per-voxel FOD can now be obtained using standard techniques such as Constrained Spherical Deconvolution [Tournier et al., 2007], allowing for the recovery of very sharp orientation profiles as shown in Fig. 7.

\section{Sensitivity to Diffusion Time}

Recent work has put focus on the diffusion time dependence of the diffusion coefficient [Fieremans et al, 2016]. When this effect has an orientational dependence, it also directly affects the estimation of diffusion anisotropy. To illustrate this, we use Camino [Cook et al., 2006] to simulate the diffusion signal in two substrates consisting of parallel axons - modelled as cylinders - with gamma distributed radii. We show cross-sections of these substrates in Fig. 8. We simulate a multi-shell acquisition with two shells using b-values $\{1000,3000\} \mathrm{s} / \mathrm{mm}^{2}$ with 30 and 60 gradient directions, respectively, and one $b_{0}$ image without diffusion weighting. We set pulse length $\delta=1 \mathrm{~ms}$ and vary pulse separation $\Delta$ from $1 \mathrm{~ms}$ to $40 \mathrm{~ms}$, while scaling the gradient strength to keep the b-values constant. While here we set $\delta$ constant to simplify the example, varying $\delta$ also influences the diffusion signal and its impact should not be ignored in practice [Åslund and Topgaard, 2009].

We show the progress of FA, GFA and PA on the right of Fig. 8 for both distributions. It can be seen that all metrics describe the signal becoming more anisotropic as diffusion time increases, even though different metrics report different levels of anisotropy. In all cases, distribution 1, having more densely packed, smaller axons, produces a more anisotropic signal.

Fitting just DTI to the $b=1000 \mathrm{~s} / \mathrm{mm}^{2}$ data, we show the progress of the diffusivities parallel $\left(D_{\|}\right)$and perpendicular $\left(D_{\perp}\right)$ to the cylinder axis on the left side 


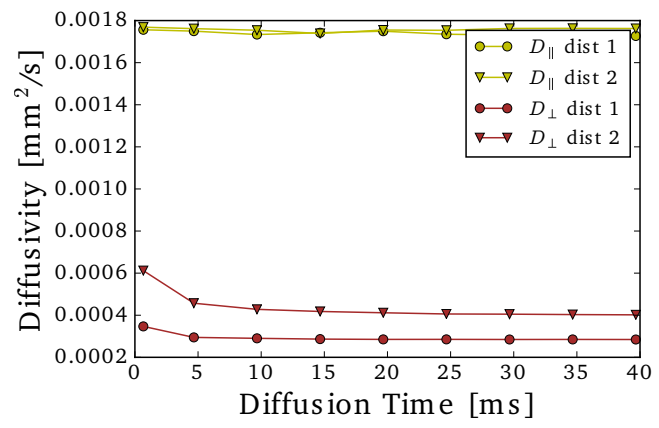

Fig. 9: The perpendicular and parallel diffusion coefficients over diffusion time for the distributions in Fig. 8.

of Fig. 9. It can be seen that time-dependence of diffusion anisotropy is caused by the time-dependence of $D_{\perp}$; lowering as diffusion time increases, indicating that diffusion is becoming more restricted. $D_{\|}$remains constant and is equal for the two distributions, indicating free parallel diffusion in this simple substrate. It should be noted that Fieremans et al. [2016] actually found $D_{\|}$to be more time-dependent than $D_{\perp}$ in-vivo, indicating that parallel diffusion is not completely free in real tissues.

\subsection{Anisotropy due to Axon Packing}

It is known that in myelinated axons, nerve conduction velocity is directly proportional to axon diameter [Waxman, 1980]. Furthermore, histology studies how that realistic axon diameters are distributed between [0.2-2] $\mu m$ [Aboitiz et al., 1992]. In this section, we continue to underline the importance of diffusion time dependence by discussing how it can be used to infer information on the axon diameter distribution and axon packing. The first model to exploit diffusion time dependence in this way was the composite hindered and restricted model of diffusion (CHARMED) model [Assaf et al., 2004]. CHARMED models axons as impermeable, parallel cylinders with fixed diameter distribution, meaning only the intra-axonal volume fraction was estimated, but not the axon diameter distribution itself. CHARMED was later extended as AxCaliber [Assaf et al., 2008] to actually estimate the gamma distribution of axon diameters. To do this, AxCaliber requires measurements exactly perpendicular to the axon direction, for different gradient strengths and diffusion times, and fits an intra-axonal and an extra-axonal tissue compartment

$$
E\left(q_{\perp}, \tau\right)=v_{\mathrm{r}} E_{\mathrm{r}}\left(q_{\perp}, \tau \mid \alpha, \beta\right)+\left(1-v_{\mathrm{r}}\right) E_{\mathrm{h}}\left(q_{\perp}, \tau\right)
$$

where $v_{\mathrm{r}}$ is the restricted water volume fraction, $E_{\mathrm{r}}\left(q_{\perp}, \tau \mid \alpha, \beta\right)$ is the perpendicular diffusion signal of cylinders [Callaghan, 1995] with Gamma distribution parameters 

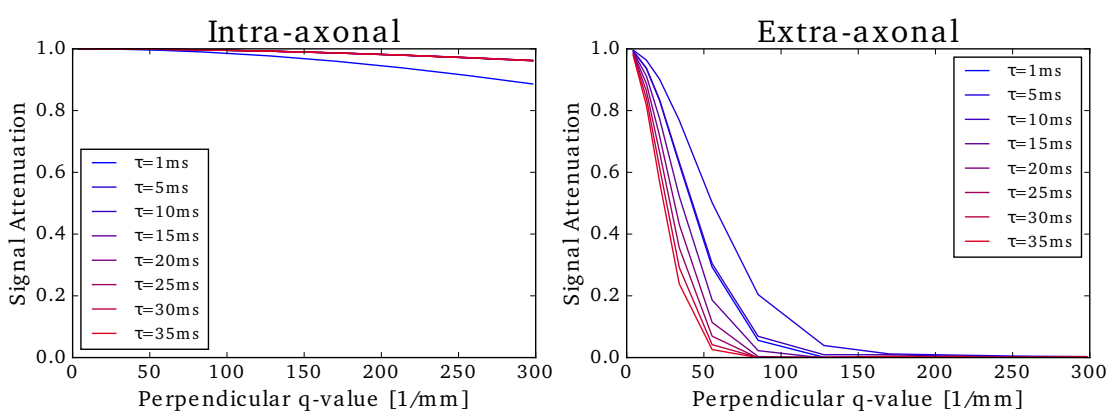

Fig. 10: The perpendicular intra-axonal and extra-axonal signal attenuation for distribution 1 of Fig. 8 for different diffusion times. It can be seen that the intra-axonal signal only shows sensitivity to diffusion time between $1 \mathrm{~ms}$ and $5 \mathrm{~ms}$, which is barely possible to achieve in practice. On the other hand, the extra-axonal signal shows a lot of contrast over this dimension.

$\alpha, \beta$ and $E_{\mathrm{h}}\left(q_{\perp}, \tau \mid D_{\mathrm{h}}\right)$ is a Gaussian with diffusivity $D_{\mathrm{h}}$ as in Eq. (7). However, there are two reasons why this model has significant limitations:

- When modeling the time-dependence of the intra-axonal signal for physically feasible ranges, the signal is basically already restricted for the shortest diffusion times, see Fig. 10.

- The non-neglible axon dispersion present in any bundle prevents the assumption that only the perpendicular direction is measured [Leergaard et al., 2010, Ronen et al., 2014].

This means that most signal variation over $\tau$ actually originates from restriction in the extra-axonal space. This behavior has recently been characterized in terms of structural disorder of the axon packing [Novikov et al., 2014, Burcaw et al., 2015]. In fact, this diffusion time dependence of the extra-axonal space has recently been implemented into the AxCaliber framework to improve axon diameter estimation [De Santis et al., 2016]. In this work, $E_{h}$ in Eq. (23) was replaced with

$$
E_{h}(\mathbf{q}, \tau)=e^{-4 \pi^{2} \tau\|\mathbf{q}\|^{2} \mathbf{g}^{T} \mathbf{D}_{h} \mathbf{g}}
$$

where $\mathbf{D}_{h}$ is made axon-packing dependent as

$$
\mathbf{D}_{h}=\left(\begin{array}{ccc}
D_{h, \|} & 0 & 0 \\
0 & D_{h, \infty}+A \frac{\ln (\Delta / \delta)+3 / 2}{\tau} & 0 \\
0 & 0 & D_{h, \infty}+A \frac{\ln (\Delta / \delta)+3 / 2}{\tau}
\end{array}\right)
$$

where $D_{h, \infty}$ is the bulk diffusion constant and A is a characteristic coefficient that scales approximately as the square of the correlation length, which in turn is proportional to the outer axonal diameter (which includes the myelin sheath) [Burcaw 
et al., 2015]. However, this augmentation does not correct for the dispersion, nor the already restricted intra-axonal diffusion at short diffusion times.

Further evidence of structural disorder was recently found in-vivo by investigating stimulated echo diffusion tensor imaging for diffusion times up to $600 \mathrm{~ms}$ [Fieremans et al., 2016]. Remarkably, both parallel and perpendicular directions show non-Gaussian diffusion for diffusion times between $45-600 \mathrm{~ms}$. This result comes after a long time of disagreement on whether or not there was time-dependence present in the nervous tissue. Hopefully, this finding will cast new insights on the interpretation of DTI studies using clinical diffusion times $\tau>20 \mathrm{~ms}$. With this in mind, it is highly likely that new scalar indices will soon be proposed to describe the amount of structural disorder as a new type of tissue biomarker.

\section{Discussion}

In this chapter, we made a specific effort to review a diffusion anisotropy related measures, coming from either signal-based dMRI models that estimate the EAP from the signal as a whole, or models that use a multi-compartment approach to estimate axon packing or axon dispersion. We started by describing the relation between the tissue and the diffusion propagator in Section 2 and illustrated this relation schematically in Fig. 1. We then explained the details of the PGSE protocol in Section 3, where we provided the sequence explicitly in Fig. 2 and illustrated different acquisition schemes (DTI, HARDI, multi-shell and $q \tau$ ) in Fig. 4. We also clarified the effect of diffusion restriction on the observed diffusion signal attenuation in the case of cylinders in Fig. 3.

We then reviewed an extensive, but probably still not an exhaustive list of diffusion anisotropy measures in Section 4. We first provided the mathematical description of signal-based anisotropy measures such as the DTI-based FA and RA [Basser, 1995]; DKI-based KFA [Jensen et al., 2005]; Q-ball-based GFA [Tuch, 2004] and MAP-MRI-based PA [Özarslan et al., 2013]. We also described the NODDI-based ODI [Zhang et al., 2012] in Section 4.3 and SMT-based $\mu$ FA [Kaden et al., 2015] in Section 4.4. We illustrated these metrics together qualitatively in Fig. 5; quantitatively in CSF, crossing and single bundle areas in Fig. 6; and illustrating the ODFs of these methods in Fig. 7. These comparisons were meant to illustrate the similarities in contrasts that different definitions of diffusion anisotropy provide, although their mathematical underpinnings may be different.

On signal-based anisotropy: Within the group of signal-based models, many studies have related changes in FA to a variety of pathologies, see e.g. the review by Assaf and Pasternak [2008]. Also characterizing the non-Gaussian parts of the data, DKI-based anisotropy measures have shown to be more sensitive than DTI-based ones[Cheung et al., 2009]. However, the literature shows that the more complex the estimation method and required acquisition scheme becomes, the fewer validation studies there are. For example, only a few studies have shown the potential of us- 
ing GFA [Cohen-Adad et al., 2011] or PA [Fick et al., 2016a], while large-scale comparisons like those for FA are missing.

Moreover, the typical criterium for being a biomarker is that the measure of interest should provide a statistically significant difference between healthy and diseased populations. However, care should be taken in prematurely calling a non-specific marker such as diffusion anisotropy a biomarker. As an illustration, in the particular case of Parkinson's disease, after many studies had claimed that FA could be used as a diagnostic biomarker, a systematic review of these studies actually showed that on its own, it cannot [Hirata et al., 2016]. It is likely that the non-specificity of diffusion anisotropy will continue to confound its interpretation as a biomarker for pathology.

On multi-compartment-based anisotropy: To overcome this lack of specificity, multi-compartment approaches strive to separate the signal contributions of different tissue compartments using biophysical models. However, it is important to realize that these models still describe diffusion anisotropy in some reparameterized way. For example, while NODDI is a multi-compartment model that separates the signal contributions of CSD, intra- and extra-axonal compartments, it can only describe one axon bundle using a Watson distribution with a single ODI, which is a function of concentration parameter $\kappa$. Illustrating the Watson ODFs together with the signalbased ODFs in Fig. 7, we indeed find its similarity to others, in particular DTI. Of course, ODI has a different interpretation than FA, but it is important to see how they are related. Furthermore, $\mu \mathrm{FA}$ describes the per-axon micro-environment and is theoretically insensitive to crossing or dispersed axon configurations.

On diffusion time-dependence: Then, in Section 5 we analyze the time-dependence of diffusion anisotropy and its origin. We illustrate in Fig. 8 that anisotropy (in a simulated ensemble of cylinders) is a function of diffusion time - longer diffusion time results in more perpendicular restriction, which translates to a higher anisotropy. However, this experiment was still limited by its simplicity, as with this setup we cannot replicate long-distance diffusion time dependence illustrated in-vivo by Fieremans et al. [2016].

Then, in Section 5.1 we go into some detail on the origin of the time-dependence, which is the extra-axonal space. We first show in Fig. 10 that the intra-axonal signal is already restricted before $5 \mathrm{~ms}$, whereas the typical minimum diffusion time in PGSE experiments is around 10ms. This means that, as was initially shown by Novikov et al. [2014], diffusing particles in the extra-axonal space, which were previously assumed to be Gaussian, are in fact still subject to some level of restriction due to axon packing. The differences in diffusion anisotropy over time shown in Fig. 8 must, therefore, be a result of the restriction in the extra-axonal space.

\section{Conclusion}

In this chapter, we have reviewed the inter-model variability of diffusion anisotropy estimation, both signal- and multi-compartment-based, as well as illustrated its sensitivity to especially short diffusion times. It is clear that there are many ways of 
defining diffusion anisotropy, depending on the chosen signal representation and acquisition scheme. Depending on the complexity of the devised metric, contrast differences can be observed both qualitative and quantitatively for different tissue types. Nonetheless, also great similarities can be appreciated between the different metrics.

Acknowledgements Data were provided [in part] by the Human Connectome Project, WUMinn Consortium (Principal Investigators: David Van Essen and Kamil Ugurbil; 1U54MH091657) funded by the 16 NIH Institutes and Centers that support the NIH Blueprint for Neuroscience Research; and by the McDonnell Center for Systems Neuroscience at Washington University. This work was partly supported by ANR "MOSIFAH" under ANR-13-MONU-0009-01 and the European Research Council (ERC) under the European Union's Horizon 2020 research and innovation program (ERC Advanced Grant agreement No 694665: CoBCoM). The author Marco Pizzolato expresses his thanks to Olea Medical and the Provence-Alpes-Côte d'Azur (PACA) Regional Council for providing grant and support. We thank Mauro Zucchelli for useful discussions.

\section{References}

Francisco Aboitiz, Arnold B Scheibel, Robin S Fisher, and Eran Zaidel. Fiber composition of the human corpus callosum. Brain research, 598(1):143-153, 1992.

Iman Aganj, Christophe Lenglet, Guillermo Sapiro, Essa Yacoub, Kamil Ugurbil, and Noam Harel. Reconstruction of the orientation distribution function in singleand multiple-shell q-ball imaging within constant solid angle. Magnetic Resonance in Medicine, 64(2):554-566, 2010.

Daniel C Alexander. An introduction to computational diffusion mri: the diffusion tensor and beyond. In Visualization and processing of tensor fields, pages 83-106. Springer, 2006.

Daniel C Alexander, Penny L Hubbard, Matt G Hall, Elizabeth A Moore, Maurice Ptito, Geoff JM Parker, and Tim B Dyrby. Orientationally invariant indices of axon diameter and density from diffusion mri. Neuroimage, 52(4):1374-1389, 2010.

JLR Andersson, J Xu, E Yacoub, E Auerbach, S Moeller, and K Ugurbil. A comprehensive gaussian process framework for correcting distortions and movements in diffusion images. In Proceedings of the 20th Annual Meeting of ISMRM, page 2426, 2012.

Ingrid Åslund and Daniel Topgaard. Determination of the self-diffusion coefficient of intracellular water using pgse $\mathrm{nmr}$ with variable gradient pulse length. Journal of Magnetic Resonance, 201(2):250-254, 2009.

Yaniv Assaf and Ofer Pasternak. Diffusion tensor imaging (dti)-based white matter mapping in brain research: a review. Journal of molecular neuroscience, 34(1): 51-61, 2008.

Yaniv Assaf, Raisa Z Freidlin, Gustavo K Rohde, and Peter J Basser. New modeling and experimental framework to characterize hindered and restricted water 
diffusion in brain white matter. Magnetic Resonance in Medicine, 52(5):965-978, 2004.

Yaniv Assaf, Tamar Blumenfeld-Katzir, Yossi Yovel, and Peter J Basser. Axcaliber: a method for measuring axon diameter distribution from diffusion mri. Magnetic Resonance in Medicine, 59(6):1347-1354, 2008.

Haz-Edine Assemlal, David Tschumperlé, and Luc Brun. Efficient and robust computation of pdf features from diffusion $\mathrm{mr}$ signal. Medical image analysis, 13(5): 715-729, 2009.

Alexandru V Avram, Joelle E Sarlls, Alan S Barnett, Evren Özarslan, Cibu Thomas, M Okan Irfanoglu, Elizabeth Hutchinson, Carlo Pierpaoli, and Peter J Basser. Clinical feasibility of using mean apparent propagator (map) mri to characterize brain tissue microstructure. NeuroImage, 2015.

Peter J Basser. Inferring microstructural features and the physiological state of tissues from diffusion-weighted images. NMR in Biomedicine, 8(7):333-344, 1995.

Peter J Basser. Relationships between diffusion tensor and q-space mri. Magnetic resonance in medicine, 47(2):392-397, 2002.

Peter J Basser, James Mattiello, and Denis LeBihan. Mr diffusion tensor spectroscopy and imaging. Biophysical journal, 66(1):259, 1994.

Christian Beaulieu. The basis of anisotropic water diffusion in the nervous system-a technical review. NMR in Biomedicine, 15(7-8):435-455, 2002.

TEJ Behrens, MW Woolrich, M Jenkinson, H Johansen-Berg, RG Nunes, S Clare, PM Matthews, JM Brady, and SM Smith. Characterization and propagation of uncertainty in diffusion-weighted $\mathrm{mr}$ imaging. Magnetic resonance in medicine, 50(5):1077-1088, 2003.

Lauren M Burcaw, Els Fieremans, and Dmitry S Novikov. Mesoscopic structure of neuronal tracts from time-dependent diffusion. NeuroImage, 114:18-37, 2015.

Paul T Callaghan. Principles of nuclear magnetic resonance microscopy, volume 3. Clarendon Press Oxford, 1991.

Paul T Callaghan. Pulsed-gradient spin-echo nmr for planar, cylindrical, and spherical pores under conditions of wall relaxation. Journal of magnetic resonance, Series A, 113(1):53-59, 1995.

Emmanuel Caruyer, Christophe Lenglet, Guillermo Sapiro, and Rachid Deriche. Design of multishell sampling schemes with uniform coverage in diffusion mri. Magnetic Resonance in Medicine, 69(6):1534-1540, 2013.

Matthew M Cheung, Edward S Hui, Kevin C Chan, Joseph A Helpern, Liqun Qi, and Ed X Wu. Does diffusion kurtosis imaging lead to better neural tissue characterization? a rodent brain maturation study. Neuroimage, 45(2):386-392, 2009.

Yoram Cohen and Yaniv Assaf. High b-value q-space analyzed diffusion-weighted $\mathrm{mrs}$ and mri in neuronal tissues-a technical review. NMR in Biomedicine, 15 (7-8):516-542, 2002.

Julien Cohen-Adad, MM El Mendili, Stéphane Lehéricy, PF Pradat, S Blancho, Serge Rossignol, and Habib Benali. Demyelination and degeneration in the injured human spinal cord detected with diffusion and magnetization transfer mri. Neuroimage, 55(3):1024-1033, 2011. 
PA Cook, Y Bai, SKKS Nedjati-Gilani, KK Seunarine, MG Hall, GJ Parker, and DC Alexander. Camino: open-source diffusion-mri reconstruction and processing. In 14th scientific meeting of the international society for magnetic resonance in medicine, volume 2759. Seattle WA, USA, 2006.

Silvia De Santis, Derek K Jones, and Alard Roebroeck. Including diffusion time dependence in the extra-axonal space improves in vivo estimates of axonal diameter and density in human white matter. NeuroImage, 130:91-103, 2016.

Maxime Descoteaux, Elaine Angelino, Shaun Fitzgibbons, and Rachid Deriche. Regularized, fast, and robust analytical q-ball imaging. Magnetic Resonance in Medicine, 58(3):497-510, 2007.

Maxime Descoteaux, Rachid Deriche, Denis Le Bihan, Jean-François Mangin, and Cyril Poupon. Multiple q-shell diffusion propagator imaging. Medical image analysis, 15(4):603-621, 2011.

Albert Einstein. Investigations on the Theory of the Brownian Movement. Courier Corporation, 1956.

Rutger Fick, Madelaine Daianu, Marco Pizzolato, Demian Wassermann, Russell E. Jacobs, Paul M. Thompson, Terrence Town, and Rachid Deriche. Comparison of biomarkers in transgenic alzheimer rats using multi-shell diffusion mri. In Computational Diffusion MRI. Springer, 2016a.

Rutger Fick, Alexandra Petiet, Mathieu Santin, Anne-Charlotte Philippe, Stephane Lehericy, Rachid Deriche, and Demian Wassermann. Multi-spherical diffusion mri: Exploring diffusion time using signal sparsity. In Computational Diffusion MRI. Springer, 2016b.

Rutger HJ Fick, Demian Wassermann, Emmanuel Caruyer, and Rachid Deriche. Mapl: Tissue microstructure estimation using laplacian-regularized map-mri and its application to hcp data. NeuroImage, 134:365-385, $2016 \mathrm{c}$.

Els Fieremans, Lauren M Burcaw, Hong-Hsi Lee, Gregory Lemberskiy, Jelle Veraart, and Dmitry S Novikov. In vivo observation and biophysical interpretation of time-dependent diffusion in human white matter. NeuroImage, 129:414-427, 2016.

Douglas N Greve and Bruce Fischl. Accurate and robust brain image alignment using boundary-based registration. Neuroimage, 48(1):63-72, 2009.

Erwin L Hahn. Spin echoes. Physical review, 80(4):580, 1950.

Brian Hansen and Sune Nørhøj Jespersen. Kurtosis fractional anisotropy, its contrast and estimation by proxy. Scientific reports, 6, 2016.

Fabiana CC Hirata, João R Sato, Gilson Vieira, Leandro T Lucato, Claudia C Leite, Edson Bor-Seng-Shu, Bruno F Pastorello, Maria CG Otaduy, Khallil T Chaim, Kenia R Campanholo, et al. Substantia nigra fractional anisotropy is not a diagnostic biomarker of parkinsons disease: A diagnostic performance study and meta-analysis. European Radiology, pages 1-9, 2016.

A Pasha Hosseinbor, Moo K Chung, Yu-Chien Wu, and Andrew L Alexander. Bessel fourier orientation reconstruction (bfor): An analytical diffusion propagator reconstruction for hybrid diffusion imaging and computation of q-space indices. NeuroImage, 64:650-670, 2013. 
Ileana O Jelescu, Jelle Veraart, Els Fieremans, and Dmitry S Novikov. Degeneracy in model parameter estimation for multi-compartmental diffusion in neuronal tissue. NMR in Biomedicine, 29(1):33-47, 2016.

Jens H Jensen, Joseph A Helpern, Anita Ramani, Hanzhang Lu, and Kyle Kaczynski. Diffusional kurtosis imaging: The quantification of non-gaussian water diffusion by means of magnetic resonance imaging. Magnetic Resonance in Medicine, 53(6):1432-1440, 2005.

Mark Kac. Can one hear the shape of a drum? The american mathematical monthly, 73(4):1-23, 1966.

Enrico Kaden, Thomas R Knösche, and Alfred Anwander. Parametric spherical deconvolution: inferring anatomical connectivity using diffusion $\mathrm{mr}$ imaging. $\mathrm{Neu}$ roImage, 37(2):474-488, 2007.

Enrico Kaden, Frithjof Kruggel, and Daniel C Alexander. Quantitative mapping of the per-axon diffusion coefficients in brain white matter. Magnetic resonance in medicine, 2015.

Enrico Kaden, Nathaniel D Kelm, Robert P Carson, Mark D Does, and Daniel C Alexander. Multi-compartment microscopic diffusion imaging. NeuroImage, 2016.

Jörg Kärger and Wilfried Heink. The propagator representation of molecular transport in microporous crystallites. Journal of Magnetic Resonance (1969), 51(1): $1-7,1983$.

Boris Keil, James N Blau, Stephan Biber, Philipp Hoecht, Veneta Tountcheva, Kawin Setsompop, Christina Triantafyllou, and Lawrence L Wald. A 64-channel $3 t$ array coil for accelerated brain mri. Magnetic Resonance in Medicine, 70(1): 248-258, 2013.

Denis Le Bihan and $\mathrm{E}$ Breton. Imagerie de diffusion in-vivo par résonance magnétique nucléaire. Comptes-Rendus de l'Académie des Sciences, 93(5):2734, 1985.

Denis Le Bihan, Jean-François Mangin, Cyril Poupon, Chris A Clark, Sabina Pappata, Nicolas Molko, and Hughes Chabriat. Diffusion tensor imaging: concepts and applications. Journal of magnetic resonance imaging, 13(4):534-546, 2001.

Trygve B Leergaard, Nathan S White, Alex De Crespigny, Ingeborg Bolstad, Helen D'Arceuil, Jan G Bjaalie, and Anders M Dale. Quantitative histological validation of diffusion mri fiber orientation distributions in the rat brain. 2010.

Daniel Liewald, Robert Miller, Nikos Logothetis, Hans-Joachim Wagner, and Almut Schüz. Distribution of axon diameters in cortical white matter: an electronmicroscopic study on three human brains and a macaque. Biological cybernetics, 108(5):541-557, 2014.

Klaus-Dietmar Merboldt, Wolfgang Hanicke, and Jens Frahm. Self-diffusion nmr imaging using stimulated echoes. Journal of Magnetic Resonance (1969), 64(3): 479-486, 1985.

Michael E Moseley, Yoram Cohen, J Kucharczyk, J Mintorovitch, HS Asgari, MF Wendland, J Tsuruda, and D Norman. Diffusion-weighted mr imaging of anisotropic water diffusion in cat central nervous system. Radiology, 176(2): 439-445, 1990. 
Dmitry S Novikov, Jens H Jensen, Joseph A Helpern, and Els Fieremans. Revealing mesoscopic structural universality with diffusion. Proceedings of the National Academy of Sciences, 111(14):5088-5093, 2014.

Evren Özarslan, Peter J Basser, Timothy M Shepherd, Peter E Thelwall, Baba C Vemuri, and Stephen J Blackband. Observation of anomalous diffusion in excised tissue by characterizing the diffusion-time dependence of the mr signal. Journal of Magnetic Resonance, 183(2):315-323, 2006.

Evren Özarslan, Timothy M Shepherd, Cheng Guan Koay, Stephen J Blackband, and Peter J Basser. Temporal scaling characteristics of diffusion as a new mri contrast: findings in rat hippocampus. Neuroimage, 60(2):1380-1393, 2012.

Evren Özarslan, Cheng Guan Koay, Timothy M Shepherd, Michal E Komlosh, M Okan İrfanoğlu, Carlo Pierpaoli, and Peter J Basser. Mean apparent propagator (map) mri: a novel diffusion imaging method for mapping tissue microstructure. NeuroImage, 78:16-32, 2013.

Y Rathi, O Michailovich, F Laun, K Setsompop, PE Grant, and C-F Westin. Multishell diffusion signal recovery from sparse measurements. Medical image analysis, 18(7):1143-1156, 2014.

Itamar Ronen, Matthew Budde, Ece Ercan, Jacopo Annese, Aranee Techawiboonwong, and Andrew Webb. Microstructural organization of axons in the human corpus callosum quantified by diffusion-weighted magnetic resonance spectroscopy of n-acetylaspartate and post-mortem histology. Brain Structure and Function, 219(5):1773-1785, 2014.

Kawin Setsompop, R Kimmlingen, E Eberlein, Thomas Witzel, Julien Cohen-Adad, Jennifer A McNab, Boris Keil, M Dylan Tisdall, P Hoecht, P Dietz, et al. Pushing the limits of in vivo diffusion mri for the human connectome project. Neuroimage, 80:220-233, 2013.

Jose Soares, Paulo Marques, Victor Alves, and Nuno Sousa. A hitchhiker's guide to diffusion tensor imaging. Frontiers in neuroscience, 7:31, 2013.

EO Stejskal and JE Tanner. Spin diffusion measurements: Spin echoes in the presence of a time-dependent field gradient. Journal of Chemical Physics, 42(1): 288-292, 1965.

Kyle Strimbu and Jorge A Tavel. What are biomarkers? Current Opinion in HIV and AIDS, 5(6):463, 2010.

JE Tanner. Transient diffusion in a system partitioned by permeable barriers. application to nmr measurements with a pulsed field gradient. The Journal of Chemical Physics, 69(4):1748-1754, 1978.

JE Tanner and Edward O Stejskal. Restricted self-diffusion of protons in colloidal systems by the pulsed-gradient, spin-echo method. The Journal of Chemical Physics, 49(4):1768-1777, 1968.

DG Taylor and MC Bushell. The spatial mapping of translational diffusion coefficients by the $\mathrm{nmr}$ imaging technique. Physics in medicine and biology, 30(4): $345,1985$.

J-Donald Tournier, Fernando Calamante, and Alan Connelly. Robust determination of the fibre orientation distribution in diffusion mri: non-negativity constrained super-resolved spherical deconvolution. NeuroImage, 35(4):1459-1472, 2007. 
Antonio Tristán-Vega, Carl-Fredrik Westin, and Santiago Aja-Fernández. Estimation of fiber orientation probability density functions in high angular resolution diffusion imaging. NeuroImage, 47(2):638-650, 2009.

Antonio Tristan-Vega, Carl-Fredrik Westin, and Santiago Aja-Fernandez. A new methodology for the estimation of fiber populations in the white matter of the brain with the funk-radon transform. NeuroImage, 49(2):1301-1315, 2010.

David S Tuch. Q-ball imaging. Magnetic Resonance in Medicine, 52(6):1358-1372, 2004.

David S Tuch, Timothy G Reese, Mette R Wiegell, Nikos Makris, John W Belliveau, and Van J Wedeen. High angular resolution diffusion imaging reveals intravoxel white matter fiber heterogeneity. Magnetic Resonance in Medicine, 48(4):577582, 2002.

David S Tuch, Timothy G Reese, Mette R Wiegell, and Van J Wedeen. Diffusion mri of complex neural architecture. Neuron, 40(5):885-895, 2003.

Stephen G Waxman. Determinants of conduction velocity in myelinated nerve fibers. Muscle \& nerve, 3(2):141-150, 1980.

Van J Wedeen, Patric Hagmann, Wen-Yih Isaac Tseng, Timothy G Reese, and Robert M Weisskoff. Mapping complex tissue architecture with diffusion spectrum magnetic resonance imaging. Magnetic Resonance in Medicine, 54(6): 1377-1386, 2005.

Hui Zhang, Torben Schneider, Claudia A Wheeler-Kingshott, and Daniel C Alexander. Noddi: practical in vivo neurite orientation dispersion and density imaging of the human brain. Neuroimage, 61(4):1000-1016, 2012. 